\title{
Proteomic Analysis of an Induced Pluripotent Stem Cell Model Reveals Strategies to Treat Juvenile Myelomonocytic Leukemia
}

\author{
Stella Pearson, ${ }^{\dagger, \nabla}$ Baoqiang Guo, ${ }^{\dagger, \#, \nabla}$ Andrew Pierce, ${ }^{\dagger, \nabla}{ }^{\circ}$ Narges Azadbakht, ${ }^{\dagger}$ Julie A. Brazzatti, ${ }^{\ddagger}$ \\ Stefano Patassini, ${ }^{\dagger}$ Sonia Mulero-Navarro, ${ }^{\S}$ Stefan Meyer, ${ }^{\dagger}$ Christian Flotho, ${ }^{\|}$Bruce D. Gelb, ${ }^{\perp}$ \\ and Anthony D. Whetton*, ${ }^{*},+$
} ${ }^{\dagger}$ Stem Cell and Leukaemia Proteomics Laboratory, Manchester Academic Health Science Centre, The University of Manchester,
Wolfson Molecular Imaging Centre, 27 Palatine Road, Withington, Manchester M20 3LJ, U.K.
${ }^{\ddagger}$ Stoller Biomarker Discovery Centre, Manchester Academic Health Science Centre, University of Manchester, Manchester M13
9 9Q U.K.
${ }^{\S}$ Department of Biochemistry, Universidad de Extremadura, Badajoz 06006, Spain
${ }^{\|}$Division of Pediatric Hematology and Oncology, Department of Pediatrics and Adolescent Medicine, Medical Center, Faculty of
Medicine, University of Freiburg, 79106 Freiburg, Germany
${ }^{\perp}$ The Mindich Child Health and Development Institute, Icahn School of Medicine at Mount Sinai, New York, New York 10029,
United States

\section{Supporting Information}

ABSTRACT: Juvenile myelomonocytic leukemia (JMML) is an aggressive myeloproliferative neoplasm of early childhood with a poor survival rate, thus there is a requirement for improved treatment strategies. Induced pluripotent stem cells offer the ability to model disease and develop new treatment strategies. JMML is frequently associated with mutations in PTPN11. Children with Noonan syndrome, a development disorder, have an increased incidence of JMML associated with specific germline mutations in PTPN11. We undertook a proteomic assessment of myeloid cells derived from induced pluripotent stem cells obtained from Noonan syndrome patients with PTPN11 mutations, either associated or not associated with an increased incidence of JMML. We report that the proteomic perturbations induced by the leukemia-associated PTPN11 mutations are associated with TP53 and NF-Kkb signaling. We have previously shown that MYC is involved in the differential gene expression observed in Noonan syndrome patients associated with an increased incidence of JMML. Thus, we employed drugs to target these pathways and demonstrate differential effects on clonogenic hematopoietic cells derived from Noonan syndrome patients, who develop JMML and those who do not. Further, we demonstrated these small molecular inhibitors, JQ1 and CBL0137, preferentially extinguish primitive hematopoietic cells from sporadic JMML patients as opposed to cells from healthy individuals.

KEYWORDS: Juvenile myelomonocytic leukemia, curaxin, Induced pluripotent stem cells, PTPN11, CBL0137

\section{INTRODUCTION}

Juvenile myelomonocytic leukemia (JMML) is an aggressive myeloproliferative neoplasm of early childhood with dysplastic features characterized by the overproduction of tissueinfiltrating myeloid cells. ${ }^{1,2}$ Currently, with the option of hematopoietic stem cell transplantation, the 5 year-event-free survival is approximately $50 \% .^{3}$ Treatment failure is typically due to relapsed disease or transformation to acute myeloid leukemia. ${ }^{4,5}$ Thus, there remains a clinical need for improved treatment strategies. ${ }^{3}$

The majority of JMML cases are associated with somatic gain-of-function mutations in the RAS/MAPK signaling pathway. ${ }^{6}$ Mutations in NF1, KRAS, PTPN11, and CBL genes are found in $>90 \%$ of cases of JMML ${ }^{7,8}$ but are mutually exclusive $^{6,9}$ suggesting the importance of this pathway in the pathogenesis of JMML. These mutations can result in the constitutive activation of numerous intracellular signaling pathways including Ras/MAPK, ${ }^{6} \mathrm{STAT}^{10}{ }^{10}$ and PI3K/AKT/ mTOR, ${ }^{11}$ all of which have been proposed as potential treatment targets. Preclinical studies in murine models have demonstrated in vivo activity of MEK and PI3K inhibitors in Ras-driven diseases, ${ }^{12-14}$ but the potential of kinase inhibitors in the treatment of JMML is presently unknown. However, targeting such downstream targets is a rational approach and

Received: July 23, 2019

Published: October 28, 2019 
there is currently a clinical trial (NCT 03190915) to investigate the effectiveness of the MEK inhibitor trametinib.

Around $35 \%$ of JMML patients harbor acquired mutations in PTPN11. PTPN11 encodes the proto-oncogene Src-homology tyrosine phosphatase2 (SHP-2), which regulates several biological processes including embryogenesis and hematopoietic cell development. ${ }^{15}$ Approximately, 50\% of children with Noonan syndrome (NS), an inherited developmental disorder, have germline mutations in PTPN11. ${ }^{16}$ Children with NS have an increased risk of developing JMML, which is associated with a distinct subset of PTPN11 mutations. ${ }^{17}$ Presently, the molecular mechanisms that account for these differences in mutant phenotypes are unclear. However, the mutants in NS associated with JMML, and somatic JMMLassociated mutations, have been reported to be more enzymatically active than those found in NS and may have different ligand-binding capacity. ${ }^{16,18}$ Investigation into the biological mechanism of leukemogenesis by proteomic analysis of the consequences of NS-associated mutations versus JMMLassociated NS mutations (NS/JMML) will increase our understanding of both NS and JMML and may identify novel targets for therapeutic intervention. While the Ras/ MAPK signaling is the primary pathway regulated by SHP-2, ${ }^{19}$ depending upon the cellular context, SHP-2 also regulates the JAK/STAT, ${ }^{20} \mathrm{PI} 3 \mathrm{~K} / \mathrm{AKT},{ }^{21}$ and focal adhesion kinase pathways, ${ }^{22}$ highlighting the need to undertake these studies in the correct context. We have previously described the generation and characterization of induced pluripotent stem cells (iPSCs) from fibroblasts of NS patients, who did (NS/JMML) and did not (NS) go on to develop JMML, which faithfully model leukemia progression after differentiation into myeloid cells. ${ }^{23}$ Derivation of iPSCs from noncancerous cells allows the investigation of these PTPN11 mutations in the absence of secondary or additional genomic alterations that frequently occur during leukemia progression. ${ }^{24}$ This overcomes the issue of the limited availability of primary cell material for studies of this disease.

Here, we describe the systematic proteomic investigation of iPSC-derived myeloid cells by mass spectrometry. We identify leukemia-associated PTPN11 mutation-induced perturbations in TP53, MYC, and NF- $\kappa$ b signaling. We demonstrate that an inhibitor of NF- $\kappa b$ that potentiates TP53 action has a differential effect on the colony-forming ability of NS and NS/JMML iPSC-derived hematopoietic cells. Further, we demonstrate the potential therapeutic benefit of this small molecular inhibitor in the treatment of sporadic JMML.

\section{METHODS}

\section{1. iPSC Maintenance and Differentiation}

iPSC lines were generated and characterized, as described in Mulero-Navarro et al. ${ }^{23}$ Single iPSC lines for wild-type, NS and NS/JMML were used in this study. Cells were maintained on irradiated mouse embryonic feeder cells in Dulbecco's modified Eagle's medium supplemented with $20 \%$ (v/v) knock-out serum replacement, $100 \mu \mathrm{M}$ nonessential amino acids, $2 \mathrm{mM}$ glutamine, $100 \mu \mathrm{M}$ mercaptoethanol (Sigma), and $10 \mathrm{ng} / \mathrm{mL}$ of hbFGF (Peprotech). Cells were passaged to new feeders as single-cell suspensions following dissociation with TrypLE Express (Gibco). Induction of hematopoietic cell differentiation was performed as described in the Supporting Methods. Cultures were maintained at $37{ }^{\circ} \mathrm{C}$ with $5 \% \mathrm{CO}_{2}, 5 \%$ $\mathrm{O}_{2}$, and routinely tested for mycoplasma contamination.

\subsection{Proteomic Assessment of CD33+ Cells}

Wild-type, NS and NS/JMML iPSC cells were differentiated toward hematopoietic progenitors over 14 days prior to the enrichment of CD33+ cells using ClinimACS (Miltenyi Biotec). Due to the relatively low yield of these clinically relevant progenitor cells, the three biological replicates for each cell line were pooled prior to being lysed to allow isobaric tagging using 8 channel iTRAQ reagent, nanoflow liquid chromatography, and tandem mass spectrometry, as previously described $^{25}$ and detailed in the Supporting Methods. Two 10 $\mu \mathrm{g}$ aliquots of protein from each pool were subject to iTRAQ labeling and MS analysis (as outlined in the Supporting Methods and Supporting Table 1). MS data of iTRAQ-labeled samples were searched against the Uniprot-homo sapiens 20160812 database containing 20191 sequence entries using ProteinPilot 5 software. This software normalizes the data using a bias correction algorithm based on the assumption that total protein expression is identical across all samples. Peptide ratios are also determined, and an average ratio produced for each protein allowing a $p$-value to be calculated as a measure of its statistical significance. All protein quantification ratios were checked to ensure that they had a normal distribution (Supporting Figure 1), and a protein change defined as a ratio outside the range in which $95 \%$ of protein ratios for the replicate runs was found with a $p$-value $<0.05$. The mass spectrometry proteomics data have been deposited to the ProteomeXchange Consortium via the PRIDE (http://www. ebi.ac.uk/pride/archive/) partner repository with the dataset identifier PXD014708.

\subsection{Cell Motility Assays}

Chemotaxis assays were performed using a Boyden chamber assay in a 96-well plate format (Neuroprobe), as previously described. $^{26}$ The number of CD $33+$ cells migrating in response to $200 \mathrm{ng} / \mathrm{mL}$ of CXCL12 was assessed over $6 \mathrm{~h}$.

\subsection{Colony-Forming Assays}

Details can be found in the Supporting Methods. In brief, colony-forming assays were performed by plating cells in methylcellulose complete media ( $R \& D$ systems) supplemented with $2 \mu / \mathrm{mL}$ of EPO. Primary CD34+ patient samples were plated at a density of 3000 cells $/ \mathrm{mL}$ and day 14 differentiated iPSCs at 4000 cells $/ \mathrm{mL}$. Colony formation was assessed following 7 and 14 days incubation at $37{ }^{\circ} \mathrm{C}$ in $5 \% \mathrm{CO}_{2} / 5 \%$ $\mathrm{O}_{2}$. Cell morphology was assessed by staining cells with MayGrunwald-Giemsa. To assess retention of self-renewal capacity, the resulting colonies at day 7 were replated in methylcellulose complete media. The use of the human tissue was in compliance with the ethical and legal framework of the Human Tissue Act. Experiments had ethical approval from the NRES committee of the regional NHS health research authority (14/LO/0489). Primary patient material was obtained from the European Working Group of MDS in Childhood (EWOG-MDS) studies (University of Freiburg, EK247/05). Control samples were surplus cells isolated from leukocyte cones from patients undergoing leukapheresis within the NHS Blood and Transplant Service. Written informed consent was obtained from participants or parents/guardians for all samples.

\subsection{Protein and mRNA Quantifications}

RNA was isolated using Qiagen RNeasy and qRT-PCR performed using standard protocols and data analyzed using the $2^{-\Delta \Delta \mathrm{CT}}$ method, as previously described. ${ }^{27}$ Protein 
expression levels were assessed by either western blot analysis or flow cytometry using standard protocols (antibodies used are detailed in the Supporting Table 2). Flow cytometric analysis was performed on a LSR Fortessa flow cytometer (BD Biosciences) using FlowJo software.

\section{RESULTS}

\subsection{Modeling Noonan Syndrome-Associated JMML}

We have previously reported ${ }^{23}$ the generation of human iPSC lines from skin fibroblasts of NS/JMML and NS patients with mutations in PTPN11 and controls with wild-type PTPN11 (Table 1).

\section{Table 1. PTPN11 Mutation Information for iPSC Lines}

\begin{tabular}{lllll}
\multicolumn{1}{c}{ iPSC } & \multicolumn{1}{c}{ disease } & gene & mutation & domain \\
WT & healthy & & none & \\
NS & Noonan syndrome & PTPN11 & E76D & N-SH2 \\
NS/JMML & $\begin{array}{l}\text { JMML progression from } \\
\text { Noonan syndrome }\end{array}$ & PTPN11 & G503R & PTP \\
& & & \\
\end{tabular}

The cell lines were shown to have a normal karyotype, to be pluripotent via in vivo teratoma formation, and exhibit increased ERK activation consistent with SHP-2 gain-offunction effects. ${ }^{23}$ All iPSC cell lines used were capable of hematopoietic differentiation; however, NS/JMML cells produced a significantly greater number of leukocytes
(CD45+) and specifically both myeloid (CD33+) and erythroid cells (CD235a+) compared to both NS and wildtype cells (Supporting Figure $2 \mathrm{~A}-\mathrm{D}$ ). The hematopoietic progenitor cells derived from NS/JMML iPSC lines, thus, recapitulated the principal features of JMML, including GMCSF hypersensitivity and a myeloid population that displayed increased proliferation. ${ }^{23}$ Our previous transcriptomic analysis of these cell lines ${ }^{23}$ indicated a role for MYC-induced regulation of the observed changes in gene expression despite a lack of difference in MYC mRNA expression levels between NS and NS/JMML cells (Supporting Figure 3A). This implies the possibility of post-translational regulation of protein levels in these cells, as we observed previously in JAK2 mutant-driven polycythemia vera. ${ }^{25}$ Given the fact that the perturbation was occurring post-translationally, we undertook a proteomic assessment of the cell lines.

\subsection{Proteomic Assessment}

We differentiated the iPSCs to hematopoietic cells in triplicate (Supporting Methods and Supporting Figure 3B) and isolated CD33-expressing myeloid cells. Due to the relatively low yield of these clinically relevant progenitor cells, the biological triplicates were pooled prior to being processed in duplicate. Isobaric tagging of tryptic peptides in duplicate followed by LC-MS/MS allowed the identification (false discovery rate < $1 \%)$ and relative quantification following normalization using the bias correction algorithm in ProteinPilot of 3585 proteins (Supporting Table 1). While this pragmatic approach of

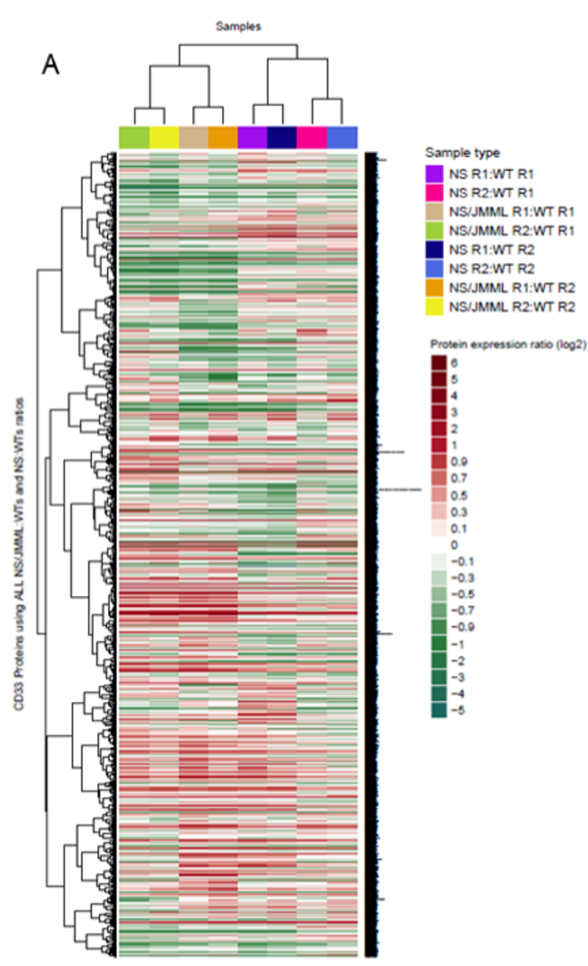

B

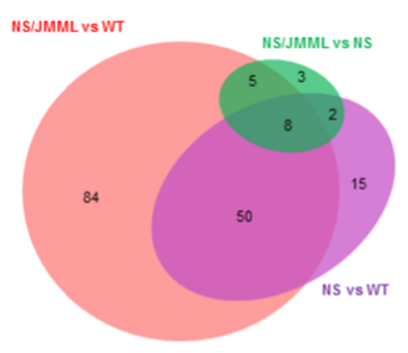

D

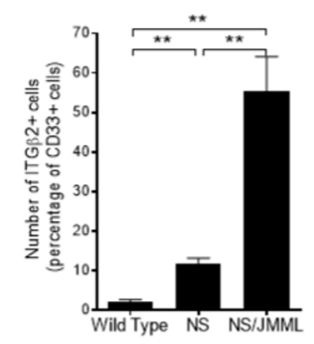

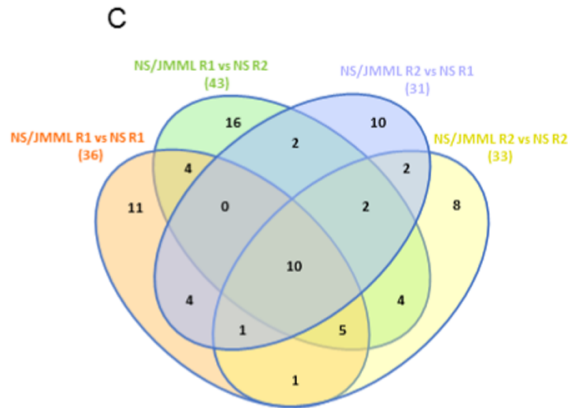

E

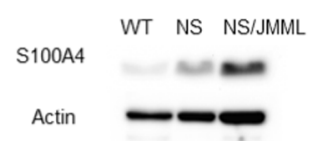

Figure 1. Proteomic analysis of CD33+ cells: (A) $\log 2$ relative protein expression ratios of the NS and NS/JMML cells with respect to the healthy control for all of the 3585 proteins identified were portrayed as an expression heatmap. Hierarchical clustering analysis on the heatmaps was computed using the average Pearson correlation method. (B) Venn diagram illustrating the relationship between the proteins identified as changing in 3 out of 4 comparisons between NS/JMML vs WT, NS vs WT, and NS/JMML vs NS. (C) Venn diagram showing the proteins identified as changing in NS vs NS/JMML in all four comparisons. To be defined as changing the protein ratio must lay outside the mean \pm two standard deviations of the duplicates with a $p$-value $<0.05$. (D) Flow cytometric assessment of ITGß2 expression in the CD33+ cells derived from WT, NS, and NS/JMML cells following 14 days culture under hematopoietic differentiation conditions. Results are shown as mean \pm scanning electron microscopy (SEM), $n=3$. Results of the $t$-test are represented by ** $p<0.01$. (E) Western blot assessment of S100A4 expression. Actin is used as a loading control (full blots in the Supporting Figure 5A). 
A

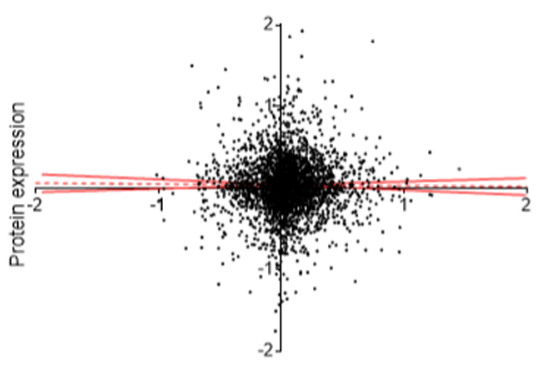

Gene expression
B

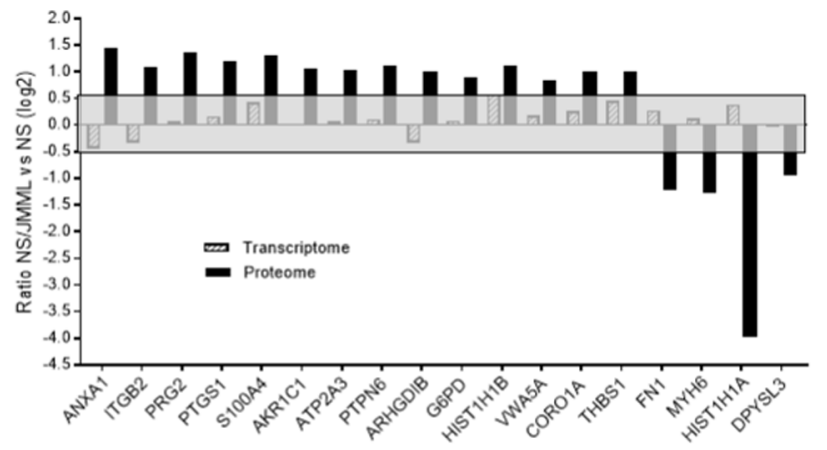

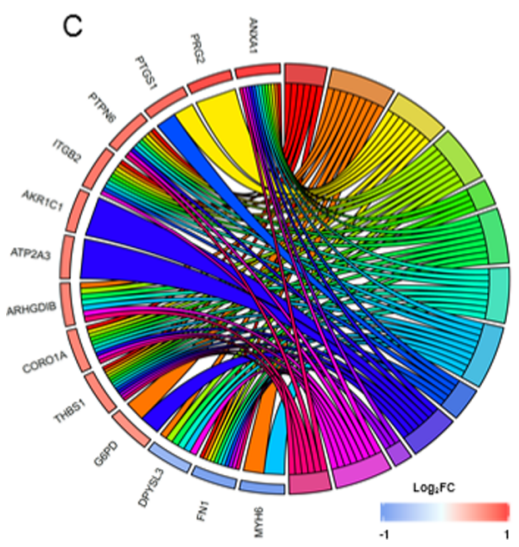

D

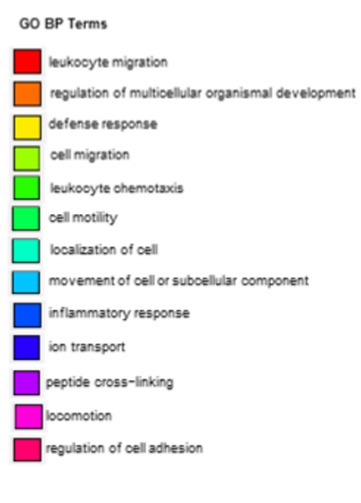

E

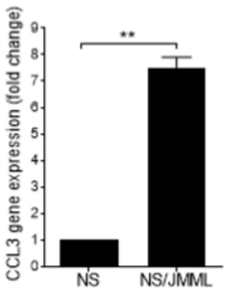

Figure 2. Analysis and validation of proteomic observations: (A) Correlation between RNA and protein expression levels. Data are shown as log 2 of the ratios between NS and NS/JMML. (B) The analysis of RNA and protein expression ratios for the 18 proteins shown to be changing in the NS/JMML vs NS comparison. Gray box encompasses the protein/gene ratios defined as not changing. (C) Gene Ontology Biological Process (GO BP) enrichment analysis was conducted using the DAVID Bioinformatics software to identify significantly enriched GO BP terms. Statistically significant results were identified as those with Benjamini-Hochberg $p$-value $<0.05$ after multiple testing correction. The statistically significant GO BP FAT category terms were then illustrated in a GOChord plot. The colors in which genes are presented reflect their log 2 -fold change as per legend. (D) The CXCL12 (200 ng/mL)-induced chemotactic response of CD33+ NS and NS/JMML was assessed in Boyden chamber assays. $5 \times$ $10^{4} \mathrm{CD} 33+$ cells (at $1 \times 10^{6}$ cells $/ \mathrm{mL}$ ) were seeded, and the number of cells migrating over a $6 \mathrm{~h}$ period counted. Results are shown as the mean number of cells migrating \pm SEM for $n=3$. (E) CCL3 expression levels were assessed by qPCR. The results are displayed as CCL3 gene expression levels in NS/JMML relative to that in NS cells (mean \pm SEM, $n=3$ ). Results of the $t$-test are represented by $* p<0.05, * * p<0.01$.

pooling has some drawbacks, it does allow the formation of suitable hypotheses that can be tested using pharmacological approaches using biological replicates. Expression analysis of the identified proteins with respect to a healthy control (WT) demonstrated significant differences between the NS and NS/ JMML cells, as shown in Figure 1A (all proteins shown). Defining a change in protein expression as a ratio outside the range in which $95 \%$ of the protein ratios for the control pools was found with a $p$-value $<0.05$ in at least three of the four replicates, 147 proteins were different in the NS/JMML vs WT comparison, 75 in the NS vs WT, and 18 in the NS/JMML vs NS (Figure 1B and Supporting Tables 3-5). Ten proteins (Supporting Table 6) were shown to change in all four comparisons between JMML and NS/JMML replicates (Figure 1C).

While we have previously demonstrated the robustness of iTRAQ data sets in multiple systems, ${ }^{25,28-31}$ we validated our data by assessing ITGß2 and S100A4 by orthogonal methods (these proteins were chosen, as validated commercial antibodies were available). Assessment of ITGß2 expression using flow cytometry (Figure 1D) and S100A4 by western blot (Figure 1E) recapitulated the iTRAQ observations (Supporting Table 2). ITGß2 is upregulated in the CD33+ cell population derived from the NS iPSCs as compared to the wild-type cells, which is further enhanced in the cells derived from the NS/JMML cell line. A similar profile of expression is seen with S100A4, which displays a large increase in expression in the NS cells that is significantly further elevated in the NS/JMML cells. Furthermore, we observed a 2-fold increase in STAT5a expression in NS/JMML cells (Supporting Table 3), which is in line with our previously published data. ${ }^{23}$ Comparison of the CD33+ cell proteomic (Supporting Table 2) and transcriptomic data ${ }^{23}$ supported our previous observations on the disparity between transcriptomic and proteomic expression. $^{32,33}$ A global analysis of the 3585 proteins (Figure $2 \mathrm{~A}$ ) showed a poor degree of correlation between mRNA and protein level changes $\left(R^{2}=4.6 \times 10^{-5}\right)$.

This is further highlighted by the fact that, of the 18 proteins shown to be differentially expressed at the protein level in the NS/JMML vs NS comparison, none of the corresponding genes showed differential expression at the mRNA level (Figure 2B). While we cannot discount that this discrepancy arises due to processes such as mRNA transport, it does stress the need for protein analysis in drug discovery and infers that post-translational regulation is mediated by PTPN11 mutations. To further validate the proteomic dataset, we undertook an in silico analysis to identify perturbed pathways. Gene ontology analysis of the proteins identified as changing between NS and NS/JMML cells using DAVID software returned leukocyte migration $\left(p=1.6 \times 10^{-5}\right)$ as the most significantly enriched biological process (Figure 2C) among several cell locomotion/migratory phenomena. We, therefore, 
A

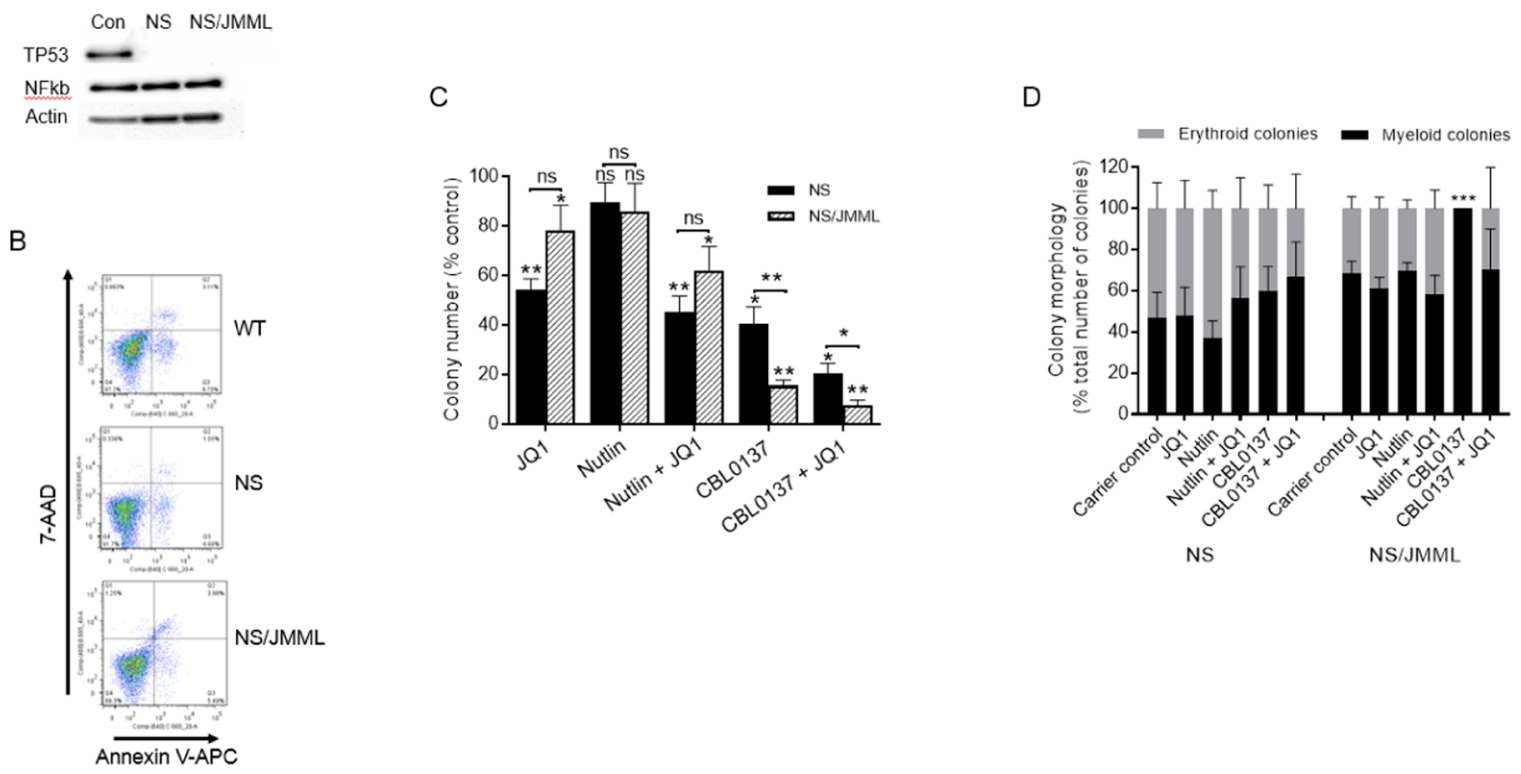

Figure 3. CBL0137 preferentially inhibits the colony-forming ability of NS/JMML cells: (A) Western blot analysis of TP53 and NF- $k \mathrm{~b}$ expression. Actin was used as a loading control (full blots in the Supporting Figure 5B). (B) The amount of apoptosis in WT, NS, and NS/JMML cells after 14 days culture under hematopoietic differentiation conditions was assessed by flow cytometry following staining with annexin V and 7-AAD. Results shown correspond to the lysates used for western blot analysis of TP53 expression in (A). (C) iPSC cells were differentiated toward hematopoietic progenitor cells, and then the effect of $250 \mathrm{nM} \mathrm{JQ1,} 100 \mathrm{nM} \mathrm{CBL0137,} \mathrm{and} 500 \mathrm{nM}$ Nutlin on their ability to form hematopoietic colonies in methylcellulose was assessed. Colonies were counted after 12 days, and the data displayed as the percentage of colonies compared to the vehicle control (mean \pm SEM, $n=8$ for NS and 9 for NS/JMML). The average number of colonies seen in the vehicle control were $35 \pm 11$ for NS and 17 \pm 4 for NS/JMML (mean \pm SEM). The results of a $t$-test between drug-treated and untreated controls and also between cell lines are shown. (D) Colony morphology was assessed and is displayed as a percentage of total colony number (mean \pm SEM, $n=4$ for NS and 5 for NS/JMML). $t$-test results are represented by; ns not significant, ${ }^{*} p<0.05, * * p<0.01, * * * p<0.001$.

investigated the ability of the differentiated NS and NS/JMML cells $(\mathrm{CD} 33+)$ to respond to CXCL12 in a Boyden chamber assay. Figure 2D illustrates the fact that CD33+ NS/JMML cells are both more motile than the NS cells and, unlike the NS cells, are able to respond to CXCL12. Dong et al. ${ }^{34}$ have recently reported that JMML-associated PTPN11 mutations lead to an increase in the production of the chemokine CCL3. We, therefore, measured the levels of CCL3 in NS and NS/ JMML cells to investigate whether differences in expression of CCL3 potentially contribute to the increased motility we observed in NS/JMML cells. NS/JMML cells display a 7-fold increase in CCL3 production (Figure 2E). This autocrine production could explain the observed difference in chemokinesis between the NS and the NS JMML cells but is unlikely to explain the differential chemotactic response to CXCL12.

The verification of the differential motility inferred by the in silico pathway analysis prompted us to undertake further in silico analysis of all of the protein changes to identify potential upstream regulators of the observed protein changes, which could constitute drug targets. Application of the Ingenuity pathway analysis software to identify key regulators driving the observed global changes in protein expression indicated a possible role for NF- $\kappa$ b and TP53 as control hubs $(p=0.0067$ and 0.0094, respectively). Western blot analysis of TP53 expression (Figure 3A) showed reduced TP53 protein levels in both NS and NS/JMML cells. To ensure that this was not simply a reflection of increased apoptosis in the control cells, programmed cell death was measured prior to cell lysis for protein measurements. All three cell lines displayed similar levels of apoptosis (Figure 3B). In contrast to TP53, NF- $\kappa$ b has a similar level of protein expression in all three cell lines
(Figure 3A). This does not rule out a role for NF- $k b$, as it is possibly the activation status of the protein rather than its gross expression level that is critical. Indeed our previous transcriptomic analysis suggests that while there is no change in NF- $\kappa$ b expression, its inhibitor NFKB1A is upregulated by 2.1 \pm 0.3 and $1.5 \pm 0.2$ fold in NS and NS/JMML cells when compared to wild-type CD33 cells.

These results implicating TP53 and NF- $\kappa \mathrm{b}$ as potential regulators of the observed protein changes along with the fact that the transcriptional analysis ${ }^{23}$ indicated a role for MYC prompted us to investigate the utility of these three pathways as targets to treat JMML.

\subsection{CBL0137 Preferentially Inhibits the Colony-Forming Ability of NS/JMML Cells}

NF- $\kappa b$, TP53, and MYC are either direct or indirect drug targets in clinical trials in other leukemias and cancers, thus targeting these pathways offers a pragmatic approach to repurposing drugs to treat JMML. We have previously demonstrated the efficiency of TP53 activation (Nutlin) in combination with MYC (JQ1) inhibition in the treatment of MPNs. ${ }^{25,28}$ Nutlin inhibits the interaction between HDM2 and TP53 leading to the stabilization of TP53. ${ }^{35} \mathrm{JQ} 1$ is a BET bromodomain inhibitor, which reduces transcription by disruption of chromatin-dependent signaling ${ }^{36}$ with MYC as a primary target. ${ }^{37}$ CBL0137 inhibits NF- $\kappa$ b, activates TP53, and has been reported to regulate MYC expression. ${ }^{38,39}$ CBL0137 is an inhibitor of the facilitates chromatin transcription complex (FACT) ${ }^{39}$ of which the component SSRP1 displays $2.7 \pm 0.4$ and $3.0 \pm 0.4$-fold increases at the transcriptome level in NS and NS/JMML cells when compared 
to wild-type CD33 cells. ${ }^{23} \mathrm{We}$, therefore, investigated the utility of these drugs to preferentially affect NS/JMML cells. Any curative treatment strategy needs to severely deplete or extinguish the primitive leukemic cells. We, therefore, investigated the effects of our candidate drugs on the ability of NS and NS/JMML iPSC-derived myeloid cells to form hematopoietic colonies. Drugs were used at optimal doses, as previously defined. ${ }^{25,28}$ Following 14 days under hematopoietic differentiation conditions, embryoid bodies were disrupted and colony-forming assays performed in the presence and absence of the drug. Colony numbers were assessed at 12 days. While all of the drug combinations, except Nutlin, had an effect on colony formation in relation to nontreated controls, CBL0137 was the only treatment with a significant differential effect on the ability of NS and NS/JMML cells to form colonies (Figure $3 \mathrm{C})$. In addition to having an enhanced inhibitory effect on NS/JMML colony number, the colonies that were formed in the presence of CBL0137 were devoid of erythroid cells (Figure 3D).

As NS patients have germline mutations in PTPN11, extinguishing mutant PTPN11 cells is obviously not a legitimate target for the treatment of NS/JMML. However, $35 \%$ of cases of sporadic JMML carry acquired mutations in PTPN11, hence CBL0137 treatment may offer the opportunity to extinguish the JMML leukemic clone stem cell while sparing healthy nonmutated PTPN11 primitive cells. Therefore, to validate our hypothesis, we transferred our iPSC observations to primary JMML cells.

\subsection{Treating Sporadic JMML with CBL0137}

To develop the paradigm, we assessed the effects of our drug combinations on CD34+ cells isolated from control nondiseased and sporadic JMML patients carrying PTPN11 mutations (Table 2).

Table 2. Genetic Information for Sporadic JMML Patients ${ }^{a}$

\begin{tabular}{cccccc} 
& & \multicolumn{3}{c}{ genetic information } \\
\cline { 3 - 6 } patient & diagnosis & gene & type & exon & mutation \\
1 & & & 3 & A72V \\
2 & & & 3 & E76K \\
3 & non-syndromic JMML & PTPN11 & somatic & 3 & E76K \\
4 & & & & 3 & E76K \\
5 & & & 3 & E76G \\
6 & & & 3 & E76K
\end{tabular}

${ }^{a}$ All patients were tested for PTPN11, NRAS, KRAS, and CBL mutations by Sanger sequencing. PTPN11 was the only mutation identified in each patient.

We first assessed the effects of our drug combinations on the ability of the CD34+ cells to form hematopoietic colonies. Treatment with Nutlin had no discernible effect on either control or JMML patient samples. Both JQ1 and CBL0137 treatment had a significant detrimental effect on colonyforming ability in sporadic JMML (Figure 4A) while having only a moderate effect on the colony-forming ability of control cells. The combination of JQ1 and CBL0137 had a marked effect on JMML cells; however, this treatment also had a significant effect on normal cells. Assessment of the colony morphology shows an increase in myeloid colonies (1.75 fold) at the expense of erythroid cell production in the JMML samples compared to controls (Figure 4B,C). As was the case in terms of colony number (Figure 4A), Nutlin had no discernible effect on the production of either erythroid or myeloid colonies (Figure 4C). JQ1 significantly reduced the number of myeloid colonies but had no effect on erythroid colony number. In contrast, CBL0137 both reduced the number of myeloid and erythroid colonies in JMML samples (Figure 4C) while having no effect on normal cell colony morphology (Figure 4B). CBL0137 effects on primary hematopoietic cells have not been determined previously, but the drug has been shown to activate apoptosis. Cells were treated in liquid culture for 3 days, and the number of cells undergoing apoptosis in different cell populations assessed by measuring annexin $\mathrm{V}$ and also CD34, CD31, CD33, and CD235a expression (Figure 5). Representative FACS plots are shown in the Supporting Figure 4. Only the JMML cells undergo significant apoptosis in the presence of CBL0137 (Figure 5A). Within the CD235a+ erythroid compartment (Figure 5B), CBL0137 can be seen to lead to the almost complete absence of any live cells in the JMML patient samples, which reflects the morphological observations (Figure 5C). CD235a-expressing cells $(62 \pm 8 \%$, mean \pm SEM) are undergoing apoptosis in the presence of CBL0137 in the JMML cells as opposed to $30 \pm 6 \%$ (mean \pm SEM) in the control cells. CBL0137 also has a differential effect between control and JMML samples on the CD34+ progenitors (Figure 5C) and CD33+ myeloid cells (Figure 5D). In both cases, CBL0137 significantly increases the degree of apoptosis in the JMML samples as compared to control cells. The only cell population, upon which CBL0137 does not have an effect on either control or JMML cells, is the CD31+ population (Figure $5 \mathrm{E})$. Given the apparent effects on different cell populations and the desire to preferentially kill primitive cells, we assessed the effects of our chosen drugs on the effective self-renewal capacity of the colony-forming cells. This was achieved by replating cells from the colonies in the first colony-forming assay (Figure 4A) into a further colony-forming assay (Figure $5 \mathrm{~F})$. While no comparison of the effects of treatment between JMML and control reached statistical significance, both CBL0137 and JQ1 treatment suggested a lowering of the replating ability in the JMML samples. Thus, using iPSC cells, we have found a means of inducing apoptosis in sporadic JMML cells for potential clinical benefit in a life-threatening disease.

\section{DISCUSSION}

Stem cell transplantation is presently the treatment of choice for JMML. However, with only a 52\% 5 year survival rate ${ }^{40}$ and allogeneic transplantation not being available worldwide, other approaches to treatment are required. While the molecular genetics of JMML has been characterized ${ }^{7,8}$ and a specific definition of the molecular pathology of the disease characterized, inclusive of GM-CSF hypersensitivity and activation of the Ras pathway, ${ }^{6}$ no targeted approach to extinguishing the leukemia clone has been identified. Hence, there is a need for an orthogonal approach to the characterization of the disease and the identification of drug targets that may offer improvements to the clinical care presently offered.

An issue in achieving this has been that JMML has proven difficult to model in vitro with the failure to generate immortalized cell lines that faithfully replicate JMML. Two methods have been successfully employed to circumvent this problem, xenotransplantation, ${ }^{4-43}$ and iPSC culture. ${ }^{23,44,45}$ The xenograft model has been used to demonstrate a potential therapeutic benefit of the DNA methyltransferase inhibitor 5- 

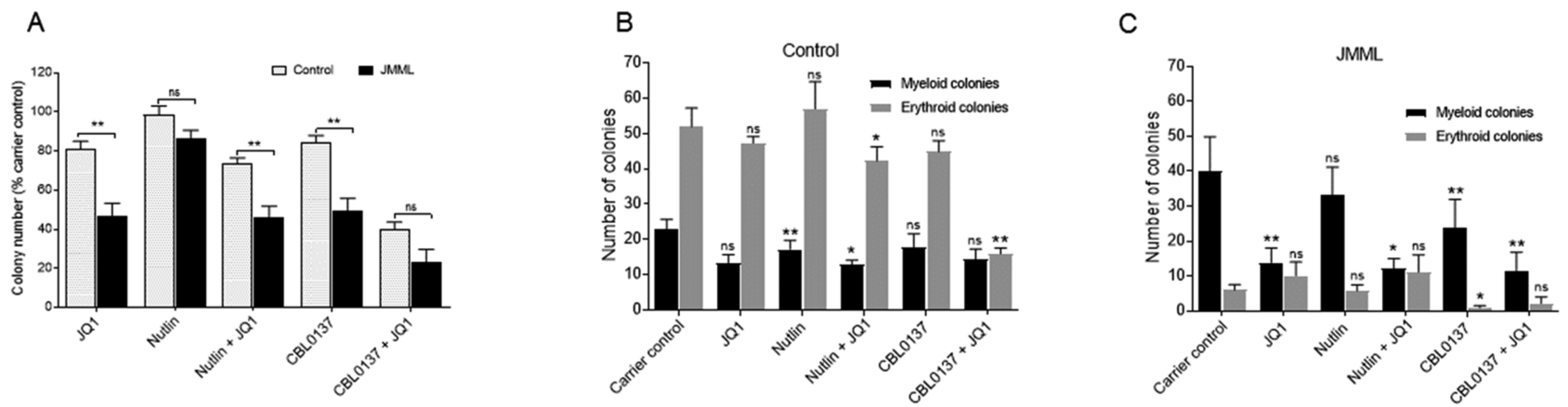

Figure 4. JQ1 and CBL0137 have a differential effect on colony-forming ability of CD34+ cells from patients with sporadic JMML: (A) The effect of $250 \mathrm{nM} \mathrm{JQ1}, 100 \mathrm{nM} \mathrm{CBL0137,} \mathrm{and} 500 \mathrm{nM}$ Nutlin on the ability of CD34+ cells from sporadic JMML patients and healthy controls to form colonies in methylcellulose was assessed. Colonies were counted after 14 days, and the data displayed as the percentage of colonies compared to the vehicle control (mean \pm SEM, $n=4$ for controls $n=6$ for JMML). The average number of colonies seen in the vehicle control was $75 \pm 4$ for controls and $46 \pm 11$ for JMML (mean \pm SEM). Colony morphology was assessed and is displayed as a percentage of total colony type (mean \pm SEM, $n=4$ for controls, $n=6$ for JMML) for control (B) and sporadic JMML (C). $t$-test results are represented by; $*^{*}<0.05, * * p<0.01, * * * p<$ 0.001 .
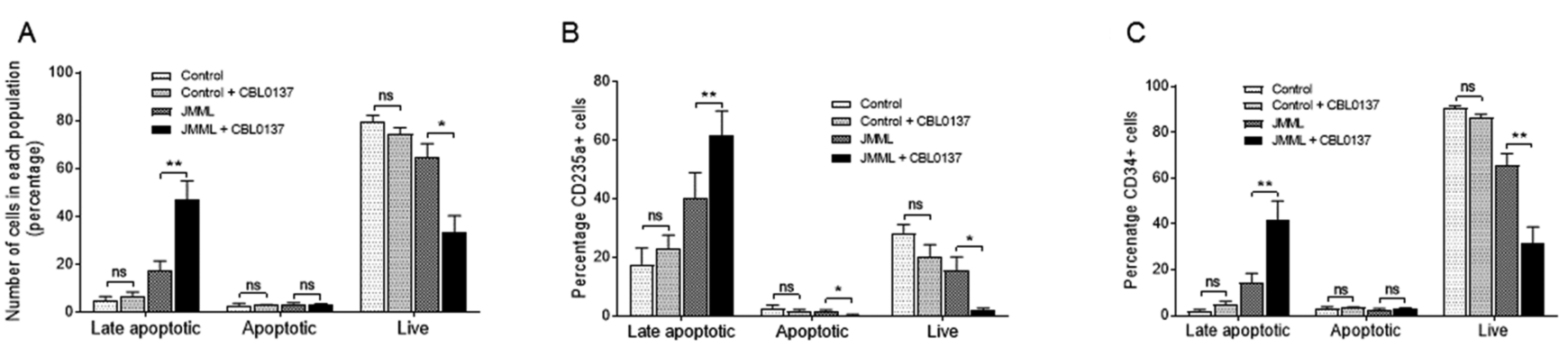

D

E

$\mathrm{F}$
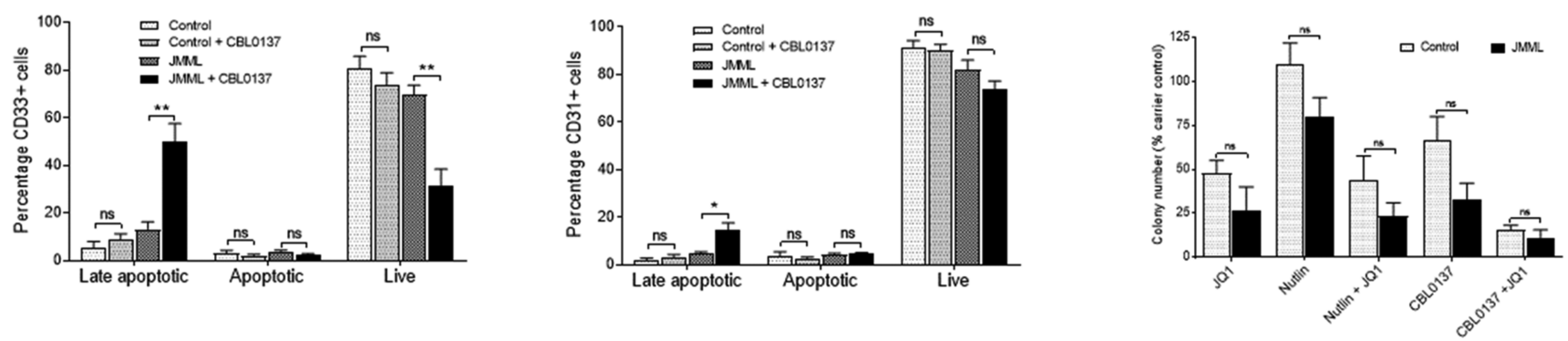

Figure 5. CBL0137 promotes apoptosis in a subset of cells: CD34+ cells isolated from control and JMML mononuclear cells were cultured for $72 \mathrm{~h}$ \pm CBL0137 (100 nM) and the degree of apoptosis measured by staining with annexin V and Hoescht (A). Cells were also counterstained with CD235a-Pacific Blue (B), CD34-PE (C), CD33-PE Cy7 (D), and CD31-FITC (E) to allow measurement of apoptosis in the separate cell populations. Cells were run on an LSR Fortessa (Becton Dickenson) and the number of live cells and those undergoing apoptosis assessed using the FlowJo software. Results are displayed as the percentage number of cells defined by each CD marker within each population (mean \pm SEM, $n=$

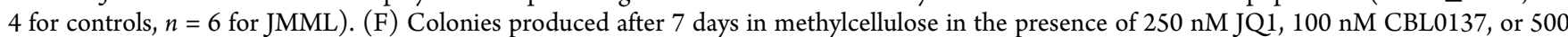
$\mathrm{nM}$ Nutlin were resuspended and replated in methylcellulose. The number of colonies was assessed following 14 days and the data expressed as a percentage of control (mean \pm SEM, $n=4$ for controls, $n=6$ for JMML). The average numbers of colonies seen in the vehicle control were $85 \pm$ 17 for controls and $27 \pm 8$ for JMML (mean \pm SEM). $t$-test results are represented by; $*_{p}<0.05, * * p<0.01$.

azacytidine. $^{46}$ Recently, an iPSC model comparing PTPN11 and $C B L$-driven JMML demonstrated differential sensitivity to MEK and JAK2 inhibition, respectively. ${ }^{45}$ Furthermore, they reported beneficial effects of simultaneous inhibition of these pathways along with the PI3K/AKT/mTOR pathway. iPSC generation from individuals with specific syndromes offers an opportunity to study their disease in an appropriate and specific cellular context. In sporadic JMML, around 35\% of patients carry a mutation in PTPN11, which is also mutated in around $50 \%$ of NS patients. ${ }^{16}$ A subset of the NS patients carrying specific germline PTPN11 mutations have an increased risk of developing JMML. ${ }^{17}$ The study on NS hematopoietic cells derived from NS/JMML (appropriate PTPN11 mutation) iPSCs and a control of Noonan iPSC (mutation in PTPN11 present but not predisposing to JMML) offers firm insight into drug targets for this disease. We used proteomics for this purpose, as many proteomic changes induced by myeloproliferative neoplasm associated oncogenes are not seen at the transcriptional level. ${ }^{33}$ Given the increased understanding of spliceosome regulation and oncogenesis, this 
is perhaps not surprising but is of particular importance as proteins are the targets for most drugs. Our strategy underlined this fact in that mRNA expression differences between NS/ JMML iPSC CD33+ cells and their NS (non-JMML) counterparts showed a low level of correlation and placed us in a position to identify new protein targets not discernible via transcriptomic analysis. The data derived from the proteomic analyses presented a phenotype of NS/JMML cells that were different in the expression of motility proteins, which translated into a distinct difference in motility itself in response to CXCL12. The role of this pathway (CXCL12/CD45/Src protein kinases/spliceosome complex proteins/MYC) in leukemogenesis has been elucidated by us in the past. ${ }^{25,26,47}$ Previous transcriptional analysis ${ }^{23}$ highlighted MYC as a potential regulator of the changes observed, while computational analysis of the global protein changes suggested NF- $\kappa b$ and TP53 as hubs differentially affected in NS and NS/JMML cells. TP53 is rarely mutated in JMML, ${ }^{48,49}$ which argues for translational or post-translational regulation of this nodal point in transformation processes in JMML. To explore novel means of extinguishing JMML cells, we chose JQ1, a MYC inhibitor, Nutlin, an activator of TP53 and CBL0137. CBL0137 targets the FACT complex and has been reported to activate TP53, modulate NF- $k b$, and adversely affect MYC signaling. ${ }^{50}$ CBL0137 also shows promise in extinguishing childhood acute lymphoblastic leukemia cells, ${ }^{51}$ hence, like JQ1 and Nutlin, offers opportunities for drug repurposing. The compelling data acquired on inhibition of NS/JMML iPSCderived CD33+ cells prompted us to take the appropriate combinations of drugs to primary JMML cells from patients with sporadic JMML. In other words, the strategy was to use the syndromic iPSC cells to gain insight into how we treat all JMMLs. There was some success in this approach in that every drug added as a single agent except Nutlin had a significant effect on colony formation in JMML as opposed to control cells. CBL0137 has been reported to act via activation of TP53 and inhibition of NF- $\kappa \mathrm{b},{ }^{39}$ however since we saw no differential effect of Nutlin on colony-forming ability, it can be hypothesized that CBL0137 is not mediating its effects via TP53. CBL0137 and JQ1 were the most potent and, in combination, had an additive effect. However, normal cells suffered greater inhibition in colony formation, meaning that this combination may be of lesser value in in vivo studies. In replating assays, it was clear that CBL0137 plus JQ1 had the strongest effect in extinguishing the leukemic clone however. CBL0137 apparently induces apoptosis, while JQ1 has previously been suggested to induce differentiation, ${ }^{28}$ explaining their additive effects.

\section{CONCLUSIONS}

We have shown that a discovery proteomic screen of iPSCderived from NS patients can lead one straight to targeted drug assays in rare and difficult to obtain primary cell material. Further, we demonstrated that our chosen drugs, JQ1 and CBL0137, preferentially extinguish primitive hematopoietic cells from sporadic JMML patients, as opposed to those of healthy individuals. We are now in a position to target the primitive JMML cell in further studies in in vivo models in a clinical research pathway that we have previously applied in a rapid and effective manner. ${ }^{28}$

\section{ASSOCIATED CONTENT}

\section{Supporting Information}

The Supporting Information is available free of charge on the ACS Publications website at DOI: 10.1021/acs.jproteome.9b00495.

Supporting Methods: Hematopoietic induction and differentiation of iPS cells, mass spectrometry analysis, primary cell colony-forming assays; Supporting Tables: proteins identified and quantified using 8plex iTRAQ in CD33+ cells from differentiated hiPSC lines (Table 1), antibodies used in the study (Table 2), proteins identified as changing between NS/JMML and control cells in the CD33+ cell population (Table 3 ), proteins identified as changing between NS and control cells in the CD33+ cell population (Table 4), proteins identified as changing between NS and NS/JMML cells in the CD33+ cell population (Table 5), proteins identified as changing in all four replicates between NS and NS/ JMML cells in the CD33+ cell population (Table 6); Supporting Figures: histogram showing the $(\log 2)$ distribution of protein quantification ratios for all proteins identified (Figure 1), flow cytometric assessment of CD45, CD33, and CD325 expression in wildtype, NS and NS/JMML cells (Figure 2), qRT-PCR results showing relative $c-M Y C$ expression and schematic representation of the hematopoietic differentiation protocol (Figure 3), representative FACS plots from the apoptosis assays shown in Figure 5 (Figure 4), full blots for the western blots (Figure 5) (PDF)

$\log 2$ protein expression ratios for NS/JMML vs WT, NS vs WT, and NS/JMML vs NS from CD33+ cells (XLSX)

\section{AUTHOR INFORMATION}

\section{Corresponding Author}

*E-mail: Tony.Whetton@Manchester.ac.uk. Tel: +44 (0)161 2750038.

ORCID $\odot$

Andrew Pierce: 0000-0001-5995-2469

Present Address

\#Division of Biomedicine, Manchester Metropolitan University, Manchester M1 5GD, U.K. (B.G.).

Author Contributions

${ }^{\nabla}$ S.P., B.G., and A.P. contributed equally to the work.

Author Contributions

A.D.W., J.A.B., B.G. designed the study. A.P. and A.D.W developed the study. S.P., B.G., A.P., S.P., S.M.-N. undertook the research. A.P., N.A., A.D.W. analyzed the data. C.F. and S.M. provided primary samples. A.P. and A.D.W. wrote the manuscript. All authors reviewed and edited the final manuscript.

Notes

The authors declare no competing financial interest.

\section{ACKNOWLEDGMENTS}

This work was supported by Children with Cancer UK (grant 14-166), NIHR BRC, Bloodwise (grant, 13005), and NIH (grant NHBLI R35 HL135742). CBL0137 was kindly 
provided by the Drug Discovery Unit, CRUK Manchester institute.

\section{ABBREVIATIONS}

JMML, Juvenile myelomonocytic leukemia; iPSCs, esInduced pluripotent stem cells; NS, Noonan syndrome; NS/JMML, JMML-associated NS mutations; WT, wild-type; FACT, facilitates chromatin transcription complex

\section{REFERENCES}

(1) Aricò, M.; Biondi, A.; Pui, C. H. Juvenile myelomonocytic leukemia. Blood 1997, 90, 479-488.

(2) Emanuel, P. D.; Shannon, K. M.; Castleberry, R. P. Juvenile myelomonocytic leukemia: molecular understanding and prospects for therapy. Mol. Med. Today 1996, 2, 468-475.

(3) Locatelli, F.; Niemeyer, C. M. How I treat juvenile myelomonocytic leukemia. Blood 2015, 125, 1083-1090.

(4) Flotho, C.; Kratz, C. P.; Bergstrasser, E.; Hasle, H.; Stary, J.; Trebo, M.; van den Heuvel-Eibrink, M. M.; Wojcik, D.; Zecca, M.; Locatelli, F.; Niemeyer, C. M. Genotype-phenotype correlation in cases of juvenile myelomonocytic leukemia with clonal RAS mutations. Blood 2008, 111, 966-967.

(5) Matsuda, K.; Shimada, A.; Yoshida, N.; Ogawa, A.; Watanabe, A.; Yajima, S.; Iizuka, S.; Koike, K.; Yanai, F.; Kawasaki, K.; Yanagimachi, M.; Kikuchi, A.; Ohtsuka, Y.; Hidaka, E.; Yamauchi, K.; Tanaka, M.; Yanagisawa, R.; Nakazawa, Y.; Shiohara, M.; Manabe, A.; Kojima, S.; Koike, K. Spontaneous improvement of hematologic abnormalities in patients having juvenile myelomonocytic leukemia with specific RAS mutations. Blood 2007, 109, 5477-5480.

(6) Yoshida, N.; Yagasaki, H.; Xu, Y.; Matsuda, K.; Yoshimi, A.; Takahashi, Y.; Hama, A.; Nishio, N.; Muramatsu, H.; Watanabe, N.; Matsumoto, K.; Kato, K.; Ueyama, J.; Inada, H.; Goto, H.; Yabe, M.; Kudo, K.; Mimaya, J.; Kikuchi, A.; Manabe, A.; Koike, K.; Kojima, S. Correlation of Clinical Features With the Mutational Status of GMCSF Signaling Pathway-Related Genes in Juvenile Myelomonocytic Leukemia. Pediatr. Res. 2009, 65, 334-340.

(7) Loh, M. L. Recent advances in the pathogenesis and treatment of juvenile myelomonocytic leukaemia. Br. J. Haematol. 2011, 152, 677687.

(8) Niemeyer, C. M.; Kratz, C. P. Paediatric myelodysplastic syndromes and juvenile myelomonocytic leukaemia: molecular classification and treatment options. Br. J. Haematol. 2008, 140, 610-624.

(9) Pérez, B.; Mechinaud, F.; Galambrun, C.; Ben Romdhane, N.; Isidor, B.; Philip, N.; Derain-Court, J.; Cassinat, B.; Lachenaud, J.; Kaltenbach, S.; Salmon, A.; Désirée, C.; Pereira, S.; Menot, M. L.; Royer, N.; Fenneteau, O.; Baruchel, A.; Chomienne, C.; Verloes, A.; Cavé, $\mathrm{H}$. Germline mutations of the CBL gene define a new genetic syndrome with predisposition to juvenile myelomonocytic leukaemia. J. Med. Genet. 2010, 47, 686-691.

(10) Kotecha, N.; Flores, N. J.; Irish, J. M.; Simonds, E. F.; Sakai, D. S.; Archambeault, S.; Diaz-Flores, E.; Coram, M.; Shannon, K. M.; Nolan, G. P.; Loh, M. L. Single-Cell Profiling Identifies Aberrant STAT5 Activation in Myeloid Malignancies with Specific Clinical and Biologic Correlates. Cancer Cell 2008, 14, 335-343.

(11) Goodwin, C. B.; Li, X. J.; Mali, R. S.; Chan, G.; Kang, M.; Liu, Z.; Vanhaesebroeck, B.; Neel, B. G.; Loh, M. L.; Lannutti, B. J.; Kapur, R.; Chan, R. J. PI3K p110delta uniquely promotes gain-offunction Shp2-induced GM-CSF hypersensitivity in a model of JMML. Blood 2014, 123, 2838-2842.

(12) Akutagawa, J.; Huang, T. Q.; Epstein, I.; Chang, T.; Quirindongo-Crespo, M.; Cottonham, C. L.; Dail, M.; Slusher, B. S.; Friedman, L. S.; Sampath, D.; Braun, B. S. Targeting the PI3K/Akt pathway in murine MDS/MPN driven by hyperactive Ras. Leukemia 2016, 30, 1335-1343.

(13) Chang, T.; Krisman, K.; Theobald, E. H.; Xu, J.; Akutagawa, J.; Lauchle, J. O.; Kogan, S.; Braun, B. S.; Shannon, K. Sustained MEK inhibition abrogates myeloproliferative disease in Nf1 mutant mice. J. Clin. Invest. 2013, 123, 335-339.

(14) Lauchle, J. O.; Kim, D.; Le, D. T.; Akagi, K.; Crone, M.; Krisman, K.; Warner, K.; Bonifas, J. M.; Li, Q.; Coakley, K. M.; DiazFlores, E.; Gorman, M.; Przybranowski, S.; Tran, M.; Kogan, S. C.; Roose, J. P.; Copeland, N. G.; Jenkins, N. A.; Parada, L.; Wolff, L.; Sebolt-Leopold, J.; Shannon, K. Response and resistance to MEK inhibition in leukaemias initiated by hyperactive Ras. Nature 2009, $461,411-414$.

(15) Nabinger, S. C.; Chan, R. J. Shp2 function in hematopoietic stem cell biology and leukemogenesis. Curr. Opin. Hematol. 2012, 19, 273-279.

(16) Tartaglia, M.; Martinelli, S.; Stella, L.; Bocchinfuso, G.; Flex, E.; Cordeddu, V.; Zampino, G.; van der Burgt, I.; Palleschi, A.; Petrucci, T. C.; Sorcini, M.; Schoch, C.; Foà, R.; Emanuel, P. D.; Gelb, B. D. Diversity and Functional Consequences of Germline and Somatic PTPN11 Mutations in Human Disease. Am. J. Hum. Genet. 2006, 78, 279-290.

(17) Mohi, M. G.; Williams, I. R.; Dearolf, C. R.; Chan, G.; Kutok, J. L.; Cohen, S.; Morgan, K.; Boulton, C.; Shigematsu, H.; Keilhack, H.; Akashi, K.; Gilliland, D. G.; Neel, B. G. Prognostic, therapeutic, and mechanistic implications of a mouse model of leukemia evoked by Shp2 (PTPN11) mutations. Cancer Cell 2005, 7, 179-191.

(18) Araki, T.; Mohi, M. G.; Ismat, F. A.; Bronson, R. T.; Williams, I. R.; Kutok, J. L.; Yang, W.; Pao, L. I.; Gilliland, D. G.; Epstein, J. A.; Neel, B. G. Mouse model of Noonan syndrome reveals cell type- and gene dosage-dependent effects of Ptpn11 mutation. Nat. Med. 2004, 10, 849-857.

(19) Neel, B. G.; Gu, H.; Pao, L. The 'Shp'ing news: SH2 domaincontaining tyrosine phosphatases in cell signaling. Trends Biochem. Sci. 2003, 28, 284-293.

(20) Qu, C. K. Role of the SHP-2 tyrosine phosphatase in cytokineinduced signaling and cellular response. Biochim. Biophys. Acta, Mol. Cell Res. 2002, 1592, 297-301.

(21) Ivins Zito, C.; Kontaridis, M. I.; Fornaro, M.; Feng, G. S.; Bennett, A. M. SHP-2 regulates the phosphatidylinositide $3^{\prime}$-kinase/ Akt pathway and suppresses caspase 3-mediated apoptosis. J. Cell. Physiol. 2004, 199, 227-236.

(22) Oh, E. S.; Gu, H.; Saxton, T. M.; Timms, J. F.; Hausdorff, S.; Frevert, E. U.; Kahn, B. B.; Pawson, T.; Neel, B. G.; Thomas, S. M. Regulation of early events in integrin signaling by protein tyrosine phosphatase SHP-2. Mol. Cell. Biol. 1999, 19, 3205-3215.

(23) Mulero-Navarro, S.; Sevilla, A.; Roman, A. C.; Lee, D. F.; D’Souza, S. L.; Pardo, S.; Riess, I.; Su, J.; Cohen, N.; Schaniel, C.; Rodriguez, N. A.; Baccarini, A.; Brown, B. D.; Cave, H.; Caye, A.; Strullu, M.; Yalcin, S.; Park, C. Y.; Dhandapany, P. S.; Yongchao, G.; Edelmann, L.; Bahieg, S.; Raynal, P.; Flex, E.; Tartaglia, M.; Moore, K. A.; Lemischka, I. R.; Gelb, B. D. Myeloid Dysregulation in a Human Induced Pluripotent Stem Cell Model of PTPN11-Associated Juvenile Myelomonocytic Leukemia. Cell Rep. 2015, 13, 504-515.

(24) Kotini, A. G.; Chang, C.-J.; Boussaad, I.; Delrow, J. J.; Dolezal, E. K.; Nagulapally, A. B.; Perna, F.; Fishbein, G. A.; Klimek, V. M.; Hawkins, R. D.; Huangfu, D.; Murry, C. E.; Graubert, T.; Nimer, S. D.; Papapetrou, E. P. Functional analysis of a chromosomal deletion associated with myelodysplastic syndromes using isogenic human induced pluripotent stem cells. Nat. Biotechnol. 2015, 33, 646-655.

(25) Pearson, S.; Williamson, A. J. K.; Blance, R.; Somervaille, T. C. P.; Taylor, S.; Azadbakht, N.; Whetton, A. D.; Pierce, A. Proteomic analysis of JAK2V617F-induced changes identifies potential new combinatorial therapeutic approaches. Leukemia 2017, 31, 27172725.

(26) Whetton, A. D.; Azmi, N. C.; Pearson, S.; Jaworska, E.; Zhang, L.; Blance, R.; Kendall, A. C.; Nicolaou, A.; Taylor, S.; Williamson, A. J.; Pierce, A. MPL W515L expression induces TGFbeta secretion and leads to an increase in chemokinesis via phosphorylation of THOC5. Oncotarget 2016, 7, 10739-10755.

(27) Guo, B.; Yang, N.; Borysiewicz, E.; Dudek, M.; Williams, J. L.; Li, J.; Maywood, E. S.; Adamson, A.; Hastings, M. H.; Bateman, J. F.; White, M. R.; Boot-Handford, R. P.; Meng, Q. J. Catabolic cytokines 
disrupt the circadian clock and the expression of clock-controlled genes in cartilage via an NFsmall ka, CyrillicB-dependent pathway. Osteoarthritis Cartilage 2015, 23, 1981-1988.

(28) Abraham, S. A.; Hopcroft, L. E. M.; Carrick, E.; Drotar, M. E.; Dunn, K.; Williamson, A. J. K.; Korfi, K.; Baquero, P.; Park, L. E.; Scott, M. T.; Pellicano, F.; Pierce, A.; Copland, M.; Nourse, C.; Grimmond, S. M.; Vetrie, D.; Whetton, A. D.; Holyoake, T. L. Dual targeting of p53 and c-MYC selectively eliminates leukaemic stem cells. Nature 2016, 534, 341-346.

(29) Holley, R. J.; Tai, G.; Williamson, A. J.; Taylor, S.; Cain, S. A.; Richardson, S. M.; Merry, C. L.; Whetton, A. D.; Kielty, C. M.; Canfield, A. E. Comparative quantification of the surfaceome of human multipotent mesenchymal progenitor cells. Stem Cell Rep. 2015, 4, 473-488.

(30) Pierce, A.; Unwin, R. D.; Evans, C. A.; Griffiths, S.; Carney, L.; Zhang, L.; Jaworska, E.; Lee, C. F.; Blinco, D.; Okoniewski, M. J.; Miller, C. J.; Bitton, D. A.; Spooncer, E.; Whetton, A. D. Eightchannel iTRAQ enables comparison of the activity of six leukemogenic tyrosine kinases. Mol Cell Proteomics 2008, 7, 853-863. (31) Unwin, R. D.; Smith, D. L.; Blinco, D.; Wilson, C. L.; Miller, C. J.; Evans, C. A.; Jaworska, E.; Baldwin, S. A.; Barnes, K.; Pierce, A.; Spooncer, E.; Whetton, A. D. Quantitative proteomics reveals posttranslational control as a regulatory factor in primary hematopoietic stem cells. Blood 2006, 107, 4687-4694.

(32) Lu, R.; Markowetz, F.; Unwin, R. D.; Leek, J. T.; Airoldi, E. M.; MacArthur, B. D.; Lachmann, A.; Rozov, R.; Ma'ayan, A.; Boyer, L. A.; Troyanskaya, O. G.; Whetton, A. D.; Lemischka, I. R. Systemslevel dynamic analyses of fate change in murine embryonic stem cells. Nature 2009, 462, 358-362.

(33) Pierce, A.; Williamson, A.; Jaworska, E.; Griffiths, J. R.; Taylor, S.; Walker, M.; Aspinall-O’Dea, M.; Spooncer, E.; Unwin, R. D.; Poolman, T.; Ray, D.; Whetton, A. D. Identification of nuclear protein targets for six leukemogenic tyrosine kinases governed by posttranslational regulation. PLoS One 2012, 7, No. e38928.

(34) Dong, L.; Yu, W.-M.; Zheng, H.; Loh, M. L.; Bunting, S. T.; Pauly, M.; Huang, G.; Zhou, M.; Broxmeyer, H. E.; Scadden, D. T.; $\mathrm{Qu}, \mathrm{C}$.-K. Leukaemogenic effects of Ptpn11 activating mutations in the stem cell microenvironment. Nature 2016, 539, 304-308.

(35) Vassilev, L. T.; Vu, B. T.; Graves, B.; Carvajal, D.; Podlaski, F.; Filipovic, Z.; Kong, N.; Kammlott, U.; Lukacs, C.; Klein, C.; Fotouhi, N.; Liu, E. A. In Vivo Activation of the p53 Pathway by SmallMolecule Antagonists of MDM2. Science 2004, 303, 844-848.

(36) Zuber, J.; Shi, J. W.; Wang, E.; Rappaport, A. R.; Herrmann, H.; Sison, E. A.; Magoon, D.; Qi, J.; Blatt, K.; Wunderlich, M.; Taylor, M. J.; Johns, C.; Chicas, A.; Mulloy, J. C.; Kogan, S. C.; Brown, P.; Valent, P.; Bradner, J. E.; Lowe, S. W.; Vakoc, C. R. RNAi screen identifies Brd4 as a therapeutic target in acute myeloid leukaemia. Nature 2011, 478, 524-528.

(37) Mertz, J. A.; Conery, A. R.; Bryant, B. M.; Sandy, P.; Balasubramanian, S.; Mele, D. A.; Bergeron, L.; Sims, R. J. Targeting MYC dependence in cancer by inhibiting BET bromodomains. Proc. Natl. Acad. Sci. U.S.A. 2011, 108, 16669-16674.

(38) Garcia, H.; Miecznikowski, J. C.; Safina, A.; Commane, M.; Ruusulehto, A.; Kilpinen, S.; Leach, R. W.; Attwood, K.; Li, Y.; Degan, S.; Omilian, A. R.; Guryanova, O.; Papantonopoulou, O.; Wang, J.; Buck, M.; Liu, S.; Morrison, C.; Gurova, K. V. Facilitates chromatin transcription complex is an "accelerator" of tumor transformation and potential marker and target of aggressive cancers. Cell Rep. 2013, 4, $159-173$.

(39) Gasparian, A. V.; Burkhart, C. A.; Purmal, A. A.; Brodsky, L.; Pal, M.; Saranadasa, M.; Bosykh, D. A.; Commane, M.; Guryanova, O. A.; Pal, S.; Safina, A.; Sviridov, S.; Koman, I. E.; Veith, J.; Komar, A. A.; Gudkov, A. V.; Gurova, K. V. Curaxins: anticancer compounds that simultaneously suppress NF-kappaB and activate p 53 by targeting FACT. Sci. Transl. Med. 2011, 3, No. 95ra74.

(40) Locatelli, F.; Nollke, P.; Zecca, M.; Korthof, E.; Lanino, E.; Peters, C.; Pession, A.; Kabisch, H.; Uderzo, C.; Bonfim, C. S.; Bader, P.; Dilloo, D.; Stary, J.; Fischer, A.; Revesz, T.; Fuhrer, M.; Hasle, H.; Trebo, M.; van den Heuvel-Eibrink, M. M.; Fenu, S.; Strahm, B.;
Giorgiani, G.; Bonora, M. R.; Duffner, U.; Niemeyer, C. M. Hematopoietic stem cell transplantation (HSCT) in children with juvenile myelomonocytic leukemia (JMML): results of the EWOGMDS/EBMT trial. Blood 2005, 105, 410-419.

(41) Krombholz, C. F.; Aumann, K.; Kollek, M.; Bertele, D.; Fluhr, S.; Kunze, M.; Niemeyer, C. M.; Flotho, C.; Erlacher, M. Long-term serial xenotransplantation of juvenile myelomonocytic leukemia recapitulates human disease in Rag2-/- $\gamma \mathrm{c}-/-$ mice. Haematologica 2016, 101, 597-606.

(42) Lapidot, T.; Grunberger, T.; Vormoor, J.; Estrov, Z.; Kollet, O.; Bunin, N.; Zaizov, R.; Williams, D.; Freedman, M. Identification of human juvenile chronic myelogenous leukemia stem cells capable of initiating the disease in primary and secondary SCID mice. Blood 1996, 88, 2655-2664.

(43) Yoshimi, A.; Balasis, M. E.; Vedder, A.; Feldman, K.; Ma, Y.; Zhang, H.; Lee, S. C.; Letson, C.; Niyongere, S.; Lu, S. X.; Ball, M.; Taylor, J.; Zhang, Q.; Zhao, Y.; Youssef, S.; Chung, Y. R.; Zhang, X. J.; Durham, B. H.; Yang, W.; List, A. F.; Loh, M. L.; Klimek, V.; Berger, M. F.; Stieglitz, E.; Padron, E.; Abdel-Wahab, O. Robust patientderived xenografts of MDS/MPN overlap syndromes capture the unique characteristics of CMML and JMML. Blood 2017, 130, 397407.

(44) Gandre-Babbe, S.; Paluru, P.; Aribeana, C.; Chou, S. T.; Bresolin, S.; Lu, L.; Sullivan, S. K.; Tasian, S. K.; Weng, J.; Favre, H.; Choi, J. K.; French, D. L.; Loh, M. L.; Weiss, M. J. Patient-derived induced pluripotent stem cells recapitulate hematopoietic abnormalities of juvenile myelomonocytic leukemia. Blood 2013, 121, 49254929.

(45) Tasian, S. K.; Casas, J. A.; Posocco, D.; Gandre-Babbe, S.; Gagne, A. L.; Liang, G.; Loh, M. L.; Weiss, M. J.; French, D. L.; Chou, S. T. Mutation-specific signaling profiles and kinase inhibitor sensitivities of juvenile myelomonocytic leukemia revealed by induced pluripotent stem cells. Leukemia 2019, 33, 181-190.

(46) Krombholz, C. F.; Gallego-Villar, L.; Sahoo, S. S.; Panda, P. K.; Wlodarski, M. W.; Aumann, K.; Hartmann, M.; Lipka, D. B.; Daskalakis, M.; Plass, C.; Niemeyer, C. M.; Erlacher, M.; Flotho, C. Azacitidine is effective for targeting leukemia-initiating cells in juvenile myelomonocytic leukemia. Leukemia 2019, 33, 1805-1810.

(47) Griaud, F.; Pierce, A.; Gonzalez Sanchez, M. B.; Scott, M.; Abraham, S. A.; Holyoake, T. L.; Tran, D. D.; Tamura, T.; Whetton, A. D. A pathway from leukemogenic oncogenes and stem cell chemokines to RNA processing via THOC5. Leukemia 2013, 27, 932-940.

(48) Honkaniemi, E.; Mattsson, K.; Barbany, G.; Sander, B.; Gustafsson, B. Elevated p53 protein expression; a predictor of relapse in rare chronic myeloid malignancies in children? Pediatr. Hematol. Oncol. 2014, 31, 327-339.

(49) Miyauchi, J.; Asada, M.; Tsunematsu, Y.; Kaneko, Y.; Kojima, S.; Mizutani, S. Abnormalities of the p53 gene in juvenile myelomonocytic leukaemia. Br. J. Haematol. 1999, 106, 980-986.

(50) Carter, D. R.; Murray, J.; Cheung, B. B.; Gamble, L.; Koach, J.; Tsang, J.; Sutton, S.; Kalla, H.; Syed, S.; Gifford, A. J.; Issaeva, N.; Biktasova, A.; Atmadibrata, B.; Sun, Y.; Sokolowski, N.; Ling, D.; Kim, P. Y.; Webber, H.; Clark, A.; Ruhle, M.; Liu, B.; Oberthuer, A.; Fischer, M.; Byrne, J.; Saletta, F.; Thwe, L. M.; Purmal, A.; Haderski, G.; Burkhart, C.; Speleman, F.; De Preter, K.; Beckers, A.; Ziegler, D. S.; Liu, T.; Gurova, K. V.; Gudkov, A. V.; Norris, M. D.; Haber, M.; Marshall, G. M. Therapeutic targeting of the MYC signal by inhibition of histone chaperone FACT in neuroblastoma. Sci. Transl. Med. 2015, 7, No. 312ra176.

(51) Lock, R.; Carol, H.; Maris, J. M.; Kolb, E. A.; Gorlick, R.; Reynolds, C. P.; Kang, M. H.; Keir, S. T.; Wu, J.; Purmal, A.; Gudkov, A.; Kurmashev, D.; Kurmasheva, R. T.; Houghton, P. J.; Smith, M. A. Initial testing (stage 1) of the curaxin CBL0137 by the pediatric preclinical testing program. Pediatr. Blood Cancer 2017, 64, No. e26263. 